\title{
A Novel Ferroptosis-Related IncRNAs Signature Predicts Clinical Prognosis and is Associated with Immune Landscape in Pancreatic Cancer
}

\section{Haiqin Ping}

Zhongnan Hospital of Wuhan University;Hubei AIDS Clinical Training Center

\section{Xingqing Jia}

JiNan City People's Hospital

Hengning Ke ( $\square$ kehengning@znhospital.cn )

Zhongnan Hospital of Wuhan University;Hubei AIDS Clinical Training Center; https://orcid.org/00000002-6091-3392

\section{Research Article}

Keywords: pancreatic cancer, ferroptosis, IncRNAs, immune.

Posted Date: September 20th, 2021

DOl: https://doi.org/10.21203/rs.3.rs-892296/v1

License: (9) This work is licensed under a Creative Commons Attribution 4.0 International License. Read Full License

Version of Record: A version of this preprint was published at Frontiers in Genetics on March 7th, 2022. See the published version at https://doi.org/10.3389/fgene.2022.786689. 


\section{Abstract}

Pancreatic cancer is one of the most lethal malignancies and currently therapies are severely lacking. In this study, we aimed to establish a novel ferroptosis-related IncRNAs signature to predict the prognosis of patients with pancreatic cancer and evaluate the predictive abilities of candidate IncRNAs. According to The Cancer Genome Atlas (TCGA) database, a total of 182 patients with pancreatic cancer were included in our study. Ferroptosis-related IncRNAs were screened by Pearson correlation analysis with 60 reported ferroptosis-related genes. Through univariate, least absolute shrinkage and selection operator (LASSO) regression and multivariate regression analyses, a novel signature based on five ferroptosis-related IncRNAs(ZNF236-DT, CASC8, PAN3-AS1, SH3PXD2A-AS1, LINP1) was constructed. Risk-related differentially expressed genes (DEGs) were subjected to enrichment analyses for Gene Ontology (GO), Kyoto Encyclopedia of Genes and Genomes (KEGG) pathway enrichment analysis. The results revealed that that immune cell infiltration, immune-related functions and checkpoints were factors to affect prognoisis of pancreatic cancer. In summary, we identified the prognostic ferroptosis-related IncRNAs in pancreatic cancer and these IncRNAs may serve as therapeutic targets for pancreatic cancer.

\section{Introduction}

Pancreatic cancer is one of the most lethal malignancies with a high mortality rate, and the incidence has steadily increased in the past few years. A family history of pancreatic cancer has been considered as an important risk factor for the disease[1], other risk factors include cigarette smoking, gender, advancing age, diabetes and so on. Early-stage pancreatic cancer is often asymptomatic. Symptoms usually occur after the surrounding tissues has been invaded or tumours metastasized to distant organs. As a result, the disease has already progressed to an advanced stage at the time of diagnosis[2, 3]. So far, surgery is considered the only potentially curative therapy for patients with pancreatic cancer.Unfortunately, many patients eventually experience recurrence after surgery and the impact of pancreatectomy on the quality of life and long-term outcomes is uncertain[4]. Therefore, identifying biomarkers for prognosis are of great clinical significance for patients.

Ferroptosis is a novel form of regulated cell death driven by iron-dependent lipid peroxidation[5]. Intracellular iron accumulation and lipid peroxidation are two central events in ferroptosis. Ferroptosis induced by small-molecule compounds or chemical drugs can suppress the growth of many types of tumors and improve the efficacy of chemotherapy, radiotherapy, or immunotherapy. In recent years, it has become a new strategy for treating many types of tumors including those from iron-rich tissues such as pancreas[6, 7]. Research showed that the deletion of a system $X_{C}{ }^{-}$subunit, SLC7A11, induced tumorselective ferroptosis and inhibited pancreatic ductal adenocarcinoma growth[8].

The long non-coding RNAs(IncRNAs) are a class of RNAs with a minimum length of about 200 nucleotides and with non-protein-coding ability[9]. The function of IncRNAs is not fully understood. It may be involved in the regulation of genes by affecting translational regulation, histone modifications, or posttranscriptional processes[10]. Studies have shown that IncRNAs play an important role in the regulation 
of cell cycle and cell differentiation in, and thus affect the development of tumors[11]. Research on IncRNAs has become a hot topic, but there are few studies on ferroptosis related IncRNA, especially in pancreatic cancer. In this article, we aimed to establish a novel ferroptosis-related IncRNAs (FRIncRNAs) signature to predict the prognosis of patients with pancreatic cancer and evaluate the predictive abilities of candidate IncRNAs. This study identified multiple ferroptosis-related IncRNAs as potential biomarkers for pancreatic cancer prognosis and further studies will improve current diagnosis, treatment, follow-up and prevention strategies of this disease.

\section{Materials And Methods}

\section{Dataset}

The public RNA sequencing of 182 patients and full clinical information were obtained from the GDC data portal using gdc-client (https://gdc-portal.nci.nih.gov/). The clinical characteristics including (age, gender, clinical grade and clinical stage) and survival overall survival (OS) information. Patients without survival information were excluded from further evaluation.In addition, the expression level of 60 ferroptosis-related genes were constructed from the dataset.

\section{Identification of Prognostic Ferroptosis-Related IncRNAs}

Based on the IncRNAannotation file downloaded from the GENCODE(https://www.gencodegenes.org/human/) website and Ensemble IDs, we annotated 14086lncRNAs and 19604 mRNAs according in the TCGA datdset. Pearsoncorrelation analysis was used to evaluate the ferroptosis-related IncRNA.LncRNAs with $p$ value $<0.01$ and an absolute Pearson correlation coeffcient $\geq 0.4$ or $\leq 0.4$ were selected as ferroptosis-related IncRNAs. Then, the univariate cox regression analysis was used to screen out theprognostic IncRNAs $(p<0.05)$. Prognostic score risk model based on independent prognostic ferroptosis- related LncRNAs

The risk model was constructed through the Least Absolute Shrinkage and Selection operator (LASSO) and multivariate Cox regression analysis. To improve the accuracy of the statistical model, Lasso Cox regression analysis was carried out by using the R package "glmnet". There are 10 ferroptosis-related IncRNAs were screened to construct the best prognostic model through the multivariate Cox regression analysis. The risk score was generated using the following formula:risk score $=$ ExpressionlncRNA1×CoefficientIncRNA1+ ExpressionIncRNA2× CoefficientlncRNA2+. . ExpressionIncRNAn $\times$ CoefficientIncRNAn. All patients were divided into high-risk and low-risk groups according to themedian cutoff of risk score.

\section{Survival Analysis}

The Kaplan-Meier survival method was applied to evaluate the availabilityof the prognostic model and all the statistical analyses were conducted using R language (version 4.0). In addition, the area under the ROC curve (AUC) was calculated to evaluate the prognostic accuracy and sensitivity of the model.Survival 
analysis was also performed for the five crucial IncRNAs respectivelyin the model. We divided pancreatic cancer patients into different subgroups and compared whether there were differences in risk scores among the groups.

\section{Establishment aNomogram}

Nomogram is a tool that can personally calculate the survival rate of patients with specific tumors and has great value in clinical application[12]. So, we constructed a nomogram to help clinicians conveniently use our modelto predict theoverall survival at 1,3 , and 5 years of patientswith pancreatic cancer. The nomogram is completed by use the R package "rms","Hmisc", it includes risk score,age,gender,grade and stage.

\section{Functional Enrichment Analysis}

We identified differentially expressed genes (DEGs) between low-risk and high-risk subgroups in the TCGA cohort in order to investigate potential biological functions and pathways between the two groups. All the DEGs in the study were performed for GeneOntology (GO) and Kyoto Encyclopedia of Genes andGenomes (KEGG) pathway enrichment analysis by using the clusterProfile R package, which the resultswith $p<0.05$ were considered significant.

\section{Evaluation of the Immune Landscape}

The proportion of tumor-infiltrating immune cellsbetween two risk groups were calculated by CIBERSORT, ESTIMATE, single-sample gene set enrichment analysis (ssGSEA) algorithms based on FRIncRNAssignature. The results were filtered with $p$ value $<0.05$.

Immune checkpoints are the immunosuppressive pathways that immune cells possess to regulate and control the persistence of the immune responses[13]. The up-regulation of immune checkpoint protein is also one of the mechanisms by which tumor cells evade immune responses[14]. In recent years,checkpoint blockade therapies have become a new type of cancer treatment. Thus, we investigated whether there were differences in the expression of immune checkpoint genes between the two risk groups, which aimed to investigate the potential role of FRIncRNAs signature in immune checkpoint blockade therapy.

Finally, we used a variety of currently acknowledged methods to analyze the relationship between risk and immune-cell characteristics, including XCELL, TIMER, QUANTISEQ, MCPOUNTER, EPIC, CIBERSORT$A B S$ and CIBERSORT. The relationship between the risk score and the immune infiltrated cells was performed by Spearmen correlation analysis. The correlation coefficient is bounded by 0 and the results were shown in a lollipop diagram.

\section{Results}

\section{Identification of Ferroptosis-Related IncRNAs in Pancreatic Cancer}


There are a total of 182 patients from the TCGA dataset who were identified and includedin this study. 14086 IncRNAs in the TCGA datasets were obtained by matching the InRNA annotation with the ENSEMBL ID downloaded from the "GENCODE" website. 60 ferroptosis-related genes were collected from the published literature for subsequent analysis. LncRNAs with $p$ value $<0.01$ and an absolute Pearson correlation coeffcient $\geq 0.4$ or $\leq 0.4$ were selected as ferroptosis-related IncRNAs. Among 246 ferroptosisrelated IncRNAs, 89 IncRNAs were identified as prognostic ferroptosis-related IncRNAs through the univariate cox regression analysis $(p<0.05)$. The research process is shown in Figure $1 \mathrm{~A}$ and the forest map of these 89 IncRNAs is shown in Fig 1B.

\section{Construction of the Ferroptosis-related IncRNAs Signature (FRLS) in the TCGA Dataset}

To build the FRLSfor the patients of pancreatic cancer in TCGA dataset, the above 89 prognostic ferroptosis-related IncRNAs were incorporated intothe least absolute shrinkage and selection operator (LASSO)regression firstly(Fig 1C, D). 10 ferroptosis-related IncRNAs including ZNF236-DT, AC005332.6, AC019186.1, AC087501.4, CASC8, PAN3-AS1, LINC01133, SH3PXD2A-AS1, LINP1, AC090114.2 were generated. To further optimize the results, we performed the multivariate Cox regression analysis to construct a prognostic model for OS usingthe expression level of the 10 ferroptosis-related IncRNAs.Anoptimal 5 IncRNAs (ZNF236-DT, CASC8, PAN3-AS1, SH3PXD2A-AS1, LINP1) signature and coefficient of eachwere identified(Fig 1E, F). The forest plot shows that ZNF236-DT and PAN3-AS1 are protective factors with HR (Hazard Ratio) < 1, while CASC8, SH3PXD2A-AS1 and LINP1 are risk factors with HR $>1$ (Fig 1E). The patients were further divided into high-risk group and low-riskgroup. The groups were generated by the formula mentioned in section "Materials and Methods". Principal Component Analyses (PCA) showed a significant distribution difference between high- and low-risk subgroups(Fig 1G, H).

The Kaplan-Meier survival analysis showed that the high-risk group had a lowerprobability of survivaland a shorter overall survival (OS) time than patients in the low-risk group(Fig 2A). The area under ROC curve (AUC) reached 0.733, which demonstrated that the FRLS could be used topredict OS in the TCGA cohort(Fig 2C). Risk score, survivalstatus and heatmap are plotted inFig 2B. Then, the 5 crucial IncRNAs of the FRLS were evaluated by survivalanalysis. The Kaplan-Meiersurvivalcurves confirmed the forest plot shown in Figure 1E,which means higher expressions of ZNF236-DT, PAN3-AS1 and lower expressions ofCASC8, SH3PXD2A-AS1 and LINP1 were associated with better overall survival (OS)(Fig 2D-H).

\section{Correlation Analysis Between the FRLS and Clinicopathological Features}

To further assess the prognostic efficacy of FRLS, we divided patients into various subgroups based on clinicopathological featuresand compared the levels of risk score between different groups. No risk score differences were observed between patients satisfied by gender and age(age was divided by 65 years old) (Fig $3 \mathrm{~A}, \mathrm{~B}$ ). But the results suggested that patients with the clinicopathologicalcharacteristics of grade 2 and 3, stage II have higher levels of riskscore compared with other correspondingsubgroups(Fig 3C, D). Thus, FRLS may have certain predictive value for clinicopathological features. The heatmap in Fig 3E further shows the distribution of clinicopathologicalcharacteristics and the risk groups. 
Afterward, we have used univariate and multivariate Cox analysesto identify independent prognostic factorsindicator.The results of univariate Cox and multivariate Coxregression analysis showed that only the risk score was significantlyassociated with OS in TCGA dataset(univariateCox analyses: HR=1.412, $95 \% \mathrm{Cl}=1.286-1.551, p<0.05$ )(Fig 3G). As shown in Fig 3F, the AUC value of the risk score was 0.724, higher than the AUC values of other clinicopathological factors. These results confirmed the FRLS is an independent and reliable prognostic indicatorsfor pancreatic cancer.

In summary, we constructed a nomogramusing the risk score (based on FRLS) and clinicopathological features, including age, gender, WHO grade and stage in the TCGAdataset(Fig $3 \mathrm{H})$.

\section{Functional Enrichment Analysis}

To investigate potential biological functions and pathways between the two risk subgroups, we performed functional enrichment analysis of the differentially expressed genes (DEGs) between the two groups. There are $973 \mathrm{DEGs}[\mid \log 2$ (fold change)| $>1$ and $p<0.05]$ between the low- and high-risk subgroups. Interestingly, the GO analysisrevealed that the DEGs were highly enriched in several immunerelated biological processes, including immune response-activating cell surface receptor signaling pathway, immune response-activating signal transduction, humoral immune response, lymphocyte mediated immunity, complement activation, B cell receptor signaling pathway(Fig 4A). Similarly, the KEGG analysis also showed significant enrichment of DEGs in immune-related pathways, for instance, cytokine-cytokine receptor interaction, chemokine signaling pathway, T cell receptor signaling pathway, B cell receptor signaling pathway(Fig 4B).

\section{Correlation of the Prognostic FRLS Withthe Immune Landscape}

Since the KEGG and GO analysis suggested that DEGs were enriched in immune-related functions and pathways,we further investigated the immune landscape in TCGA dataset. The ESTIMATE algorithm showed that the immunescore, stromal score and ESTIMATEscore were all higher in the low-risk group than in the high-risk group(Fig $5 A, B, C)$. These results also suggest that tumor purity was lower in the lowrisk group than in the high-risk group.

Moreover, the CIBERSORT algorithm was used to analyze the 22 different immune cell types among the two risk group. As shown in Fig5D, B cell naive, T cell CD8 and T cells regulatory(Tregs) were up-regulated in the low risk subgroup of the TCGA cohort, while NK cells activated and mast cell resting were significantly down-regulated $(p<0.05)$. Next,wefurther analyzed the correlation between FRLS and immune cells. The results indicated that this signature was most significantly positive correlation with immune infiltration of Macrophages $\mathrm{M0}$ (Cor $=0.25, p=0.004$ ), Macrophages $\mathrm{M} 1$ (Cor $=0.2, p=0.022$ ), Macrophages M2(Cor $=0.22, p=0.012$ ) and Mast cells resting(Cor $=0.2, p=0.02$ ), but negatively correlated with $B$ cells naive(Cor $=-0.35, p=4.5 \mathrm{e}-05)$, Plasma cells(Cor $=-0.2, p=0.023)$, T cells CD4 memory activated(Cor $=-0.2, p=0.022$ ), T cells CD8 (Cor=-0.3, $p=0.00059$ ) and T cells regulatory(Tregs, Cor $=-0.27$, $p=0.0014$ )(Fig $5 \mathrm{~F}-\mathrm{N})$. These findings again confirmed that this FRIncRNAs signature was associated to immune cell infiltration in pancreatic cancer. 
By using the sSGSEA analysis, we quantified the enrichment scores of immune-related functions in the two risk groups. The results showed that the high-risk group was significantly correlated with most immune-related functions, such as CCR, Check-point, Cytolytic-activity, HLA, Inflammation-promoting, T cell co-inhibition, T cell co-stimulation and Type II IFN Response(Fig 5E). Moreover, the expression of immune checkpoint molecules also differed significantlybetween the two risk groups, such as HHLA2, CD44 and TNFSF9 in the high-risk group were higher than those in the low-risk group, while other molecules were higher in the low-risk group(Fig 6A).

Finally, detailed Spearman correlation analysis was conducted. The risk score was positively correlated with common lymphoid progenitor,CD $4^{+}$T cells, Macrophage M1, Macrophage M0, Neutrophil,NK cells,Eosinophil and Monocyte.It was inversely associated with most other tumor-infiltrating immune cells(Fig 6B).

\section{Discussion}

In this study, a total of 182 patients with pancreatic cancer from the TCGA dataset were included to exploit the prognostic significance of ferrotosis-related IncRNAs. Firstly, we identified 89 prognostic ferroptosis-related IncRNAs through the univariate Cox regression analysis. Under the LASSO regression and multivariate Cox regression analysis, five IncRNAs of them were selected to establish the novel prognostic FRLS for predicting the OS of patients with pancreatic cancer. Among the five IncRNAs, ZNF236-DT and PAN3-AS1 are protective factors, while CASC8, SH3PXD2A-AS1 and LINP1 are risk factors. Secondly, based on the median risk score, patients were divided into the low- and high-risk subgroups and the high-risk group had worse clinical outcomes than patients in the low-risk group. Functional enrichment analysis showed that the DEGs between the two subgroups were highly enriched in several immune-related biological processes and pathways. Finally, we compared the immune and stromal scores, the infiltration of different immune cells and expression levels of immune checkpoint molecules. These results confirmed differences in the immune landscape between the two risk subgroups. As far as we know, this is the first study to confirm that ferroptosis-related IncRNAs could be used as biomarkers to predict the survival prognosis of patients with pancreatic cancer.

Pancreatic cancer is a malignant tumor with a high mortality rate. So far, the underlying mechanism of pancreatic cancer is not completely understood. A number of studies have revealed the functions and regulatory roles of IncRNAs in pancreatic cancer behaviors. Hisashi Yoshimura revealed that $\mathrm{H} 19$ has important roles in pancreatic cancer metastasis, and that inhibition of $\mathrm{H} 19$ represents a novel candidate for pancreatic cancer therapy[15]. SNHG15 could act as an oncogene in pancreatic cancer, further research found that SNHG15 knockdown inhibited proliferative capacities and suppressed apoptotic rate of pancreatic cancer cells in vitro, and impaired in vivo tumorigenicity[16]. However, there are few studies about ferroptosis-related IncRNAs, especially in pancreatic cancer.

In ferroptotic cells, cytological changes mainly include increased mitochondrial membrane density, decrease or disappearance of mitochondrial crest [17]. Ferroptosis is a novel method for the destruction 
of cancer cells and it can be triggered by some exogenic small molecules,e.g., erastin, Ras-selective lethal small molecule 3, certain clinical drugs and even nano ferroptosis inducers. Notably, ferroptosis has also been shown to be associated with tumor immunotherapy[18]. IncRNAs are part of the noncoding RNAs family and several new studies have suggested that IncRNAs play an important role in ferroptosis of tumors. For instance, long noncoding RNA LINC00336 was found to inhibit ferroptosis in lung cancer by functioning as a competing endogenous RNA via sponging miR-6852[19]. In addition, Mao[20] found that IncRNA P53RRA can bind to Ras GTPase-activating protein-binding protein 1 (G3BP1) and promote ferroptosis in breast cancer and lung cancer. Among the established 5 ferroptosis-related IncRNAs in this study, three have been found to be associated with tumor development. Silencing CASC8 inhibited the proliferation, migration, and invasion of non-small cell lung cancer cells and promoted their sensitivity to osimertinib[21]. LINP1 may be involved in the regulation of cell proliferation, cell adhesion and cell cyclerelated biological processes in early stage pancreatic ductal adenocarcinoma[22]. Mechanism studies have found that SH3PXD2A-AS1 can directly interact with p53 protein and regulate p53-mediated gene transcription in colorectal cancer, and eventually lead to increased cell proliferation, angiogenesis, and metastasis[23].

The DEGs between the two subgroups of the FRLS were highly enriched in several immune-related biological processes and pathways and the immune score, stromal score were significantly different between the two groups. B cells, $\mathrm{CD} 8^{+} \mathrm{T}$ cells, Tregs were significantly enriched in the low risk group, NK cells and mast cells were enriched in the high risk group. Among these types of cell, mast cells, B cells and Tregs are antigen presenting cell(APCs) that are capable of presenting processed antigens to $T$ cells and activating the immune response. Besides, it is interesting that the immune-related functions also differed significantly between the two risk groups. These functions are related to the development and treatment of tumors. For instance, Hiraoka $\mathrm{N}$ et al found that the higher expression of HLA-I, HLA-E and HLA-G on pancreatic ductal adenocarcinoma cells is an unfavorable prognosticator[24]. High cytolytic activity was confirmed to associate with increased expression of genes involved in multiple immune checkpoints (with the notable exception of PD-L1) in pancreatic cancer[25]. Therefore, we assume that ferroptosis is closely related to the immune landscape of microenvironment in pancreatic cancer. However, the potential molecular mechanisms remain to be explored by in-depth experimental researches.

In addition, further analysis found that the low-risk group exhibited higher expression levels of immune checkpoints. Among these immune checkpoints, therapy that targets programmed death 1 or programmed death 1 ligand 1 (PD-1/PD-L1) has been rapidly developing as oncotherapy for various carcinomas, including pancreatic cancer. Whatsmore, CD200 has been shown to promote immunosuppression in the pancreatic tumor microenvironment and targeting CD200 may enhance activity of checkpoint immunotherapy[26]. Although immunotherapies such as checkpoint inhibition monotherapy have yet to demonstrate efficacy, a growing body of evidence suggests that combination regimens including chemotherapy could unlock immunotherapy in pancreatic cancer. Our analysis offered the possibility of several potential checkpoint targets for pancreatic cancer, but the mechanism and efficacy remained to be validated in the future. 
However, there are several limitations in our study. Firstly, our study is only based on the TCGA public database and the sample size was relatively small. Secondly, our study lacks a validation cohort set and prospective, multicenter, real-world data to be further verified.

\section{Declarations}

Funding This work was funded by the National Natural Science Foundation of China (31760263) to Dr. Hengning Ke and the Non-profit Central Research Institute Fund of the Chinese Academy of Medical Sciences(2020-PT320-004).

Conflict of interest There are no conflicts of interest to declare.

Authors' contributions Haiqin Ping collected the data and designed the research and Xingqing Jia conducted statistical analyses. Haiqin Ping drafted the manuscript, Hengning Ke revised the manuscript. All authors collected patient data and read and approved the fnal manuscript.

Availability of data and material The data in this study are available in TCGAdatabase at https://portal.gdc.cancer.gov/.

\section{References}

1.Raimondi S, Maisonneuve P, Lowenfels AB. Epidemiology of pancreatic cancer: an overview. Nat Rev Gastroenterol Hepatol. 2009 Dec;6(12):699-708.

2.Kamisawa T, Wood LD, Itoi T, Takaori K. Pancreatic cancer. Lancet. 2016 Jul 2;388(10039):73-85.

3.Gillen S, Schuster T, Meyer Zum Büschenfelde C, Friess H, Kleeff J. Preoperative/neoadjuvant therapy in pancreatic cancer: a systematic review and meta-analysis of response and resection percentages. PLoS Med. 2010 Apr 20;7(4):e1000267.

4.Siegel R, Ma J, Zou Z, Jemal A. Cancer statistics, 2014. CA Cancer J Clin. 2014 Jan-Feb;64(1):9-29.

5.Xie Y, Hou W, Song X, Yu Y, Huang J, Sun X, Kang R, Tang D. Ferroptosis: process and function. Cell Death Differ. 2016 Mar;23(3):369-79.

6.Eling N, Reuter L, Hazin J, Hamacher-Brady A, Brady NR. Identification of artesunate as a specific activator of ferroptosis in pancreatic cancer cells. Oncoscience. 2015 May 2;2(5):517-32.

7.Xie Y, Song X, Sun X, Huang J, Zhong M, Lotze MT, Zeh HJ Rd, Kang R, Tang D. Identification of baicalein as a ferroptosis inhibitor by natural product library screening. Biochem Biophys Res Commun. 2016 May 13;473(4):775-780.

8.Badgley MA, Kremer DM, Maurer HC, DelGiorno KE, Lee HJ, Purohit V, Sagalovskiy IR, Ma A, Kapilian J, Firl CEM, Decker AR, Sastra SA, Palermo CF, Andrade LR, Sajjakulnukit P, Zhang L, Tolstyka ZP, Hirschhorn 
T, Lamb C, Liu T, Gu W, Seeley ES, Stone E, Georgiou G, Manor U, luga A, Wahl GM, Stockwell BR, Lyssiotis CA, Olive KP. Cysteine depletion induces pancreatic tumor ferroptosis in mice. Science. $2020 \mathrm{Apr}$ 3;368(6486):85-89.

9.Guttman M, Rinn JL. Modular regulatory principles of large non-coding RNAs. Nature. 2012 Feb $15 ; 482(7385): 339-46$.

10.Huang Q, Yan J, Agami R. Long non-coding RNAs in metastasis. Cancer Metastasis Rev. 2018 Mar;37(1):75-81.

11.Ramilowski, J. A., Yip, C. W., Agrawal, S., Chang, J. C., Ciani, Y., Kulakovskiy, I. V.,et al. Functional Annotation of Human Long Noncoding RNAs via Molecular Phenotyping. Genome Res. 2020 Jul;30(7):1060-1072.

12.Park SY. Nomogram: An analogue tool to deliver digital knowledge. J Thorac Cardiovasc Surg. 2018 Apr;155(4):1793.

13.Abril-Rodriguez G, Ribas A. SnapShot: Immune Checkpoint Inhibitors. Cancer Cell. 2017 Jun 12;31(6):848-848.e1.

14.Pardoll DM. The blockade of immune checkpoints in cancer immunotherapy. Nat Rev Cancer. 2012 Mar 22;12(4):252-64.

15.Yoshimura H, Matsuda Y, Yamamoto M, Michishita M, Takahashi K, Sasaki N, Ishikawa N, Aida J, Takubo K, Arai T, Ishiwata T. Reduced expression of the H19 long non-coding RNA inhibits pancreatic cancer metastasis. Lab Invest. 2018 Jun;98(6):814-824.

16.Ma Z, Huang H, Wang J, Zhou Y, Pu F, Zhao Q, Peng P, Hui B, Ji H, Wang K. Long non-coding RNA SNHG15 inhibits P15 and KLF2 expression to promote pancreatic cancer proliferation through EZH2mediated H3K27me3. Oncotarget. 2017 Aug 18;8(48):84153-84167.

17.Yagoda N, Von RM, Zaganjor E, Bauer AJ, Yang WS, Fridman DJ, et al. RAS-RAF-MEK-dependent oxidative cell death involving voltage-dependent anion channels. Nature. 2007 Jun 14;447(7146):864-8.

18.Wang W, Green M, Choi JE, et al. CD8+ T cells regulate tumour ferroptosis during cancer immunotherapy. Nature. 2019 May;569(7755):270-274.

19.Wang M, Mao C, Ouyang L, Liu Y, Lai W, Liu N, Shi Y, Chen L, Xiao D, Yu F, Wang X, Zhou H, Cao Y, Liu S, Yan Q, Tao Y, Zhang B. Long noncoding RNA LINC00336 inhibits ferroptosis in lung cancer by functioning as a competing endogenous RNA. Cell Death Differ. 2019 Nov;26(11):2329-2343.

20.Mao C, Wang X, Liu Y, Wang M, Yan B, Jiang Y, Shi Y, Shen Y, Liu X, Lai W, Yang R, Xiao D, Cheng Y, Liu S, Zhou H, Cao Y, Yu W, Muegge K, Yu H, Tao Y. A G3BP1-Interacting IncRNA Promotes Ferroptosis and Apoptosis in Cancer via Nuclear Sequestration of p53. Cancer Res. 2018 Jul 1;78(13):3484-3496. 
21.Jiang X, Guan J, Xu Y, Ren H, Jiang J, Wudu M, Wang Q, Su H, Zhang Y, Zhang B, Zou Z, Hu Y, Sun X, Qiu X. Silencing of CASC8 inhibits non-small cell lung cancer cells function and promotes sensitivity to osimertinib via FOXM1. J Cancer. 2021 Jan 1;12(2):387-396.

22.Shang LM, Liao XW, Zhu GZ, Huang KT, Han CY, Yang CK, Wang XK, Zhou X, Su H, Ye XP, Peng T. Genome-wide RNA-sequencing dataset reveals the prognostic value and potential molecular mechanisms of IncRNA in non-homologous end joining pathway 1 in early stage Pancreatic Ductal Adenocarcinoma. $J$ Cancer. 2020 Jul 20;11(19):5556-5567.

23.Hou P, Lin T, Meng S, Shi M, Chen F, Jiang T, Li Z, Li M, Chu S, Zheng J, Bai J. Long noncoding RNA SH3PXD2A-AS1 promotes colorectal cancer progression by regulating p53-mediated gene transcription. Int J Biol Sci. 2021 May 11;17(8):1979-1994.

24.Hiraoka N, Ino Y, Hori S, Yamazaki-Itoh R, Naito C, Shimasaki M, Esaki M, Nara S, Kishi Y, Shimada K, Nakamura N, Torigoe T, Heike Y. Expression of classical human leukocyte antigen class I antigens, HLA-E and HLA-G, is adversely prognostic in pancreatic cancer patients. Cancer Sci. 2020 Aug;111(8):30573070 .

25.Balli D, Rech AJ, Stanger BZ, Vonderheide RH. Immune Cytolytic Activity Stratifies Molecular Subsets of Human Pancreatic Cancer. Clin Cancer Res. 2017 Jun 15;23(12):3129-3138.

26. Choueiry F, Torok M, Shakya R, Agrawal K, Deems A, Benner B, Hinton A, Shaffer J, Blaser BW, Noonan AM, Williams TM, Dillhoff M, Conwell DL, Hart PA, Cruz-Monserrate Z, Bai XF, Carson WE 3rd, Mace TA. CD200 promotes immunosuppression in the pancreatic tumor microenvironment. J Immunother Cancer. 2020 Jun;8(1):e000189.

\section{Figures}


A

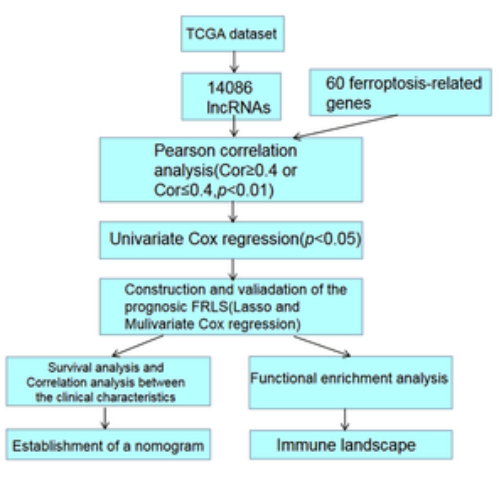

C

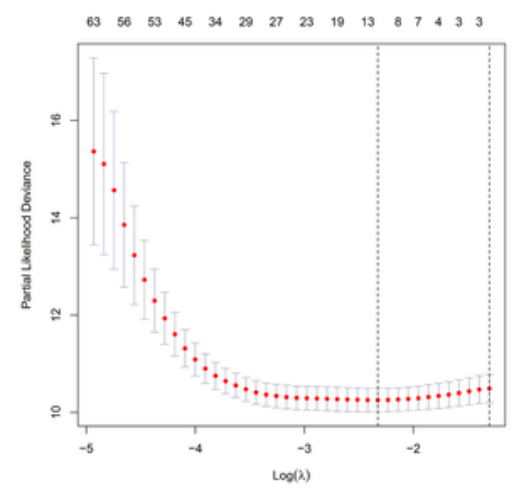

$\mathrm{E}$

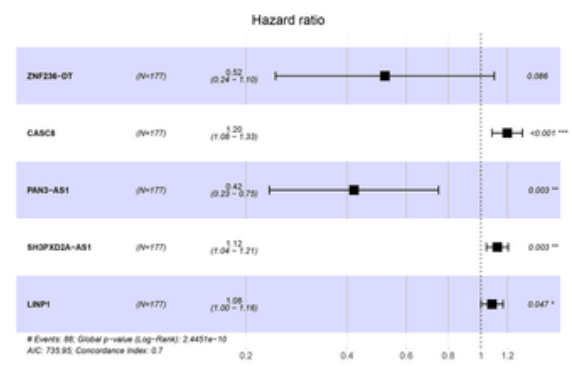

G

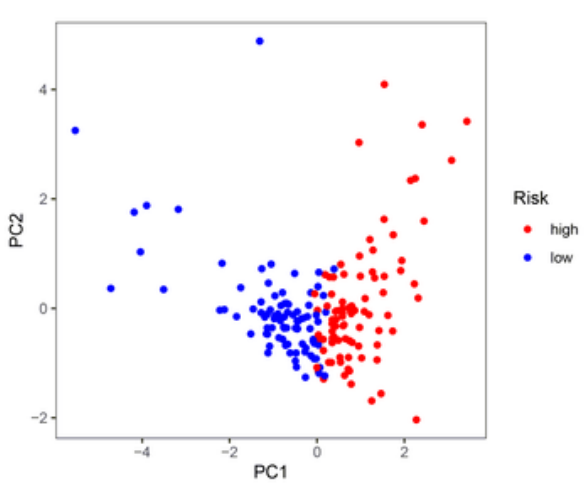

B

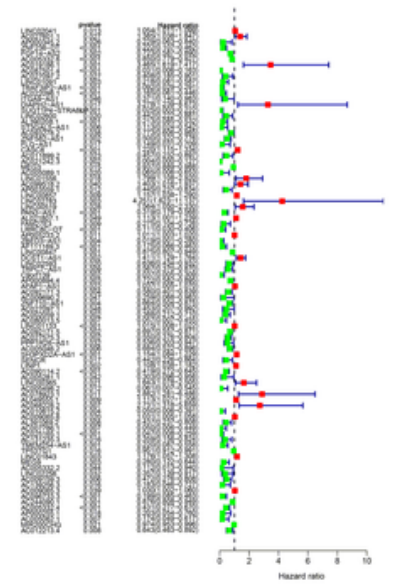

D

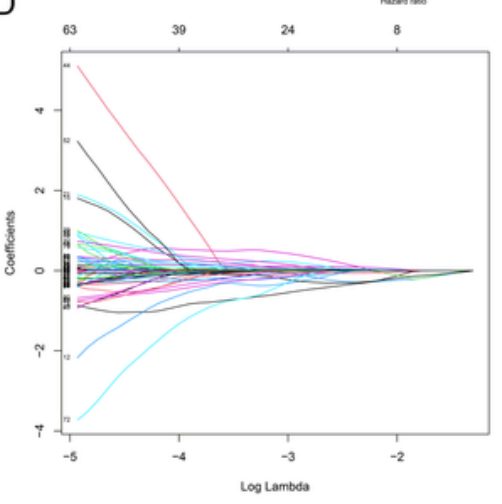

$\mathrm{F}$

\begin{tabular}{cc}
\hline Symbol & Coef \\
\hline ZNF236-DT & -0.657228039 \\
CASC8 & 0.180841017 \\
PAN3-AS1 & -0.869147432 \\
SH3PXD2A-AS1 & 0.112889411 \\
LINP1 & 0.076780468 \\
\hline
\end{tabular}

$\mathrm{H}$

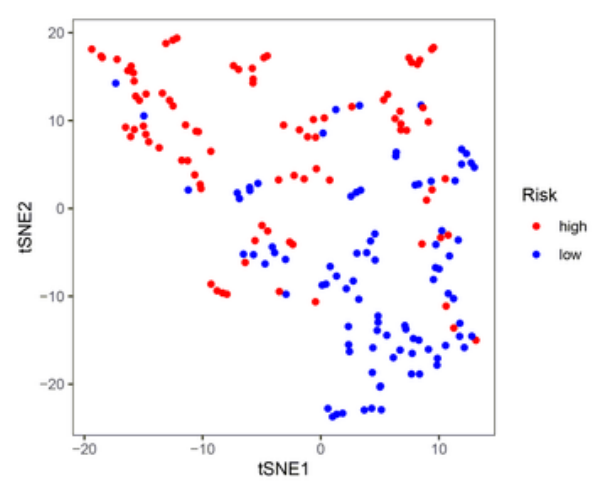

\section{Figure 1}

(A). The flow diagram of the research process. (B).Forest plots revealed prognosis-related ferroptosisrelated IncRNAs based on the results of univariate Cox regression. (C-D). The least absolute shrinkage and selection operator(LASSO) regression was performed with the minimum criteria.An optimal $\log \lambda$ value is indicated by the vertical black line in the plot. $(E)$. The forest plot of the multivariate Cox regression analysis. (F). The coefficients of five selected ferroptosis-related IncRNAs measured by the 
multivariate Cox. (G).PCA plot showing the distribution of the established FRLS expression in different risk groups. $(\mathrm{H})$.t-SNE plot showing the distribution of the patients in different risk groups.

A
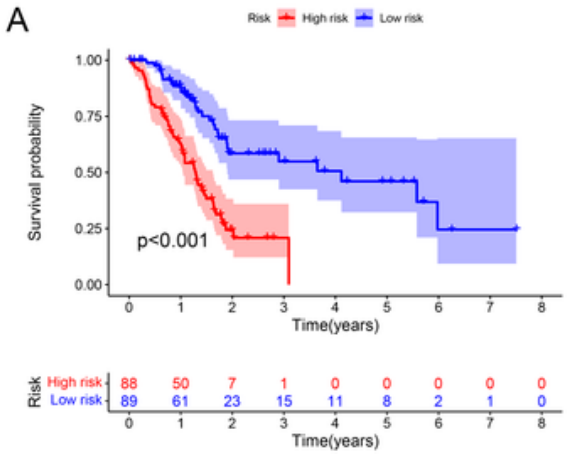

C

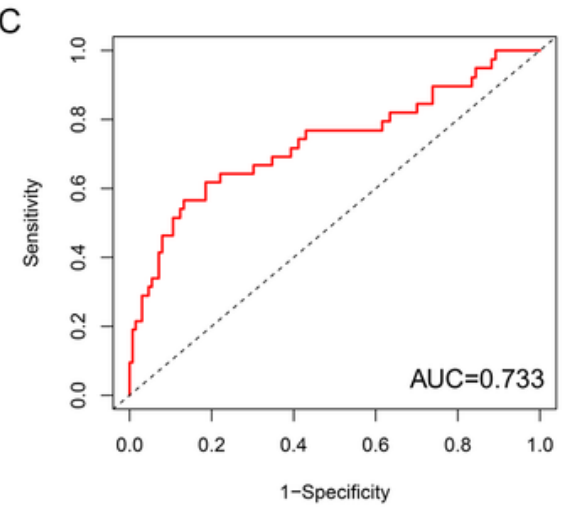

B
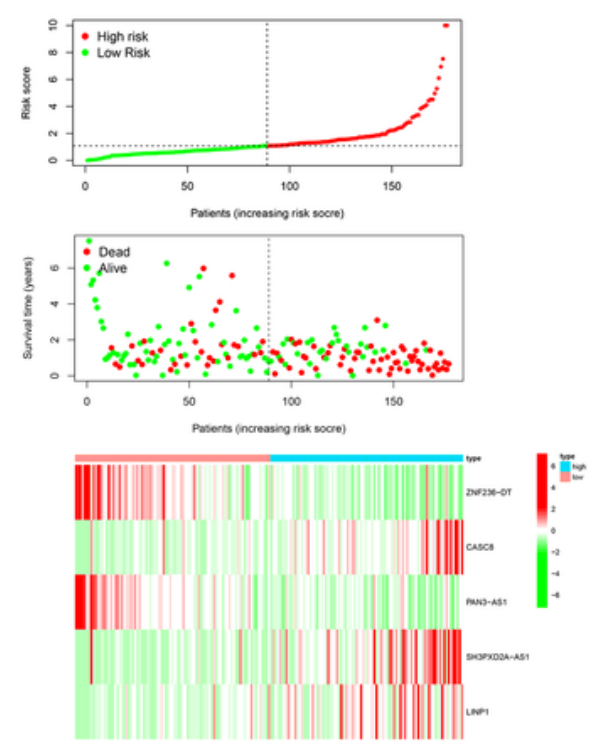

D

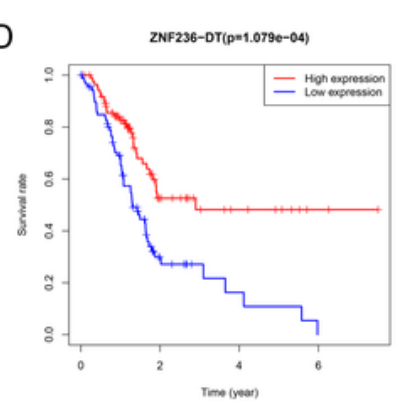

E
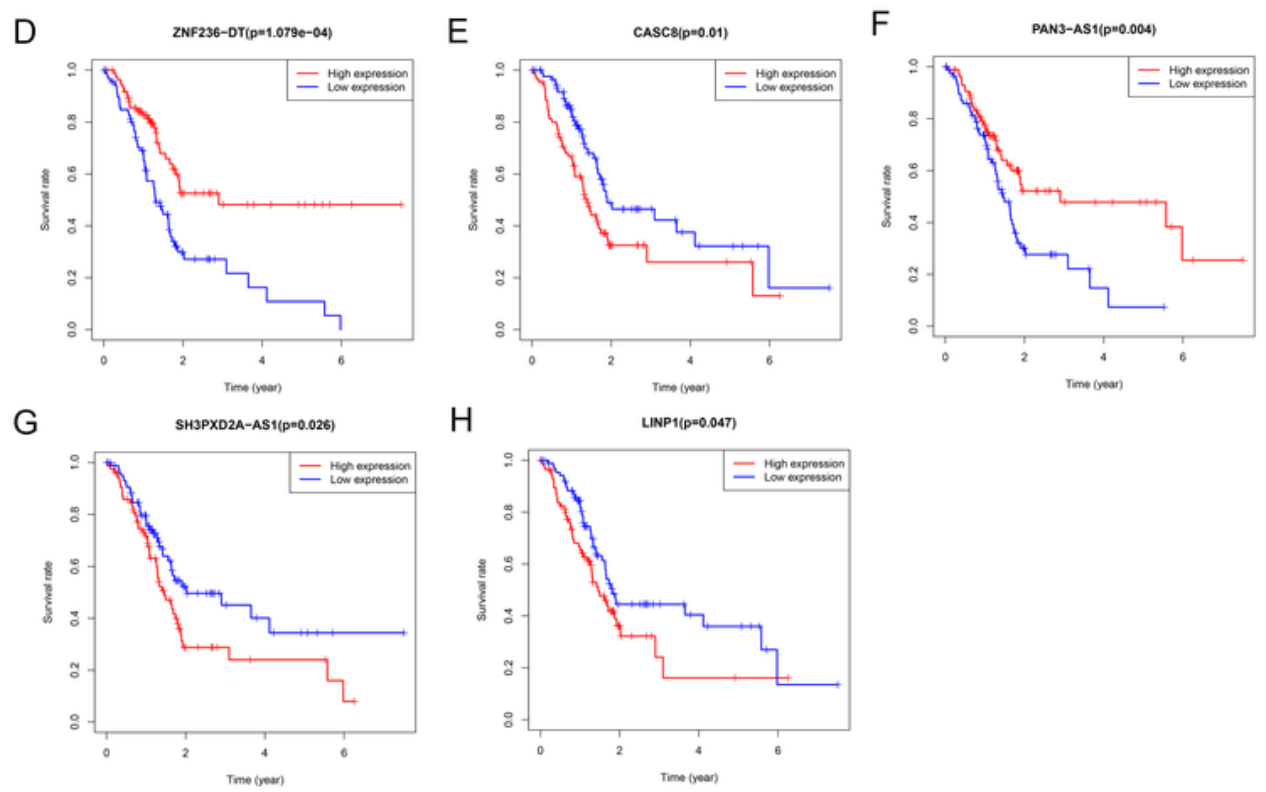

\section{Figure 2}

(A).Kaplan-Meier curves for the overall survival of patients in the high- and low-risk groups in the TCGA cohort. (B).Distributions of risk scores,survival status and expression of five ferroptosis-related IncRNAs in the TCGA dataset. (C). The receiver operating characteristic (ROC) curve analyses of the prognostic 
FRLS in the TCGA cohorts. (D-H).Kaplan-Meier curves analyzed on the correlation between the expression of the five crucial IncRNAs and the prognosis of pancreatic cancer.

A

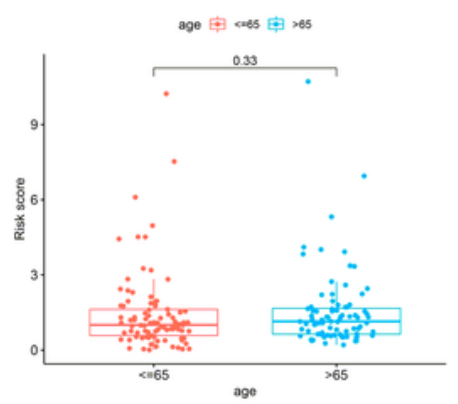

C

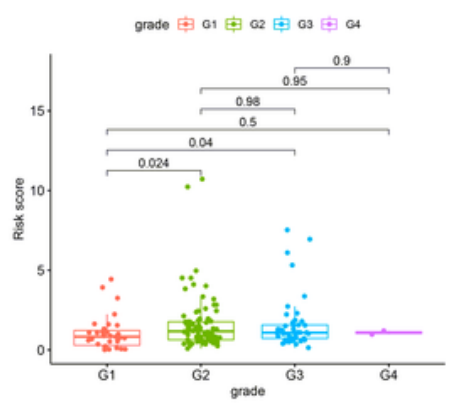

B

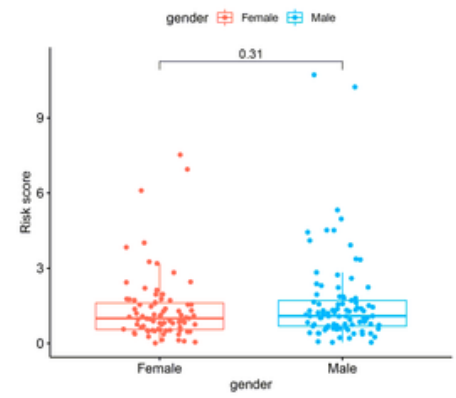

D

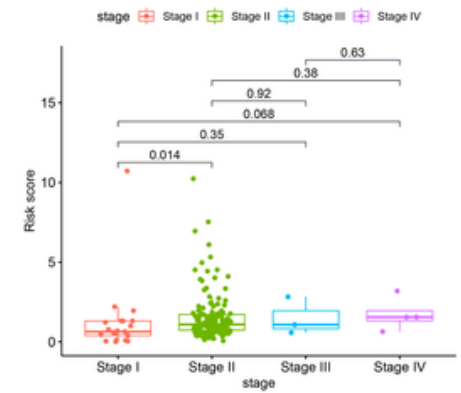

E

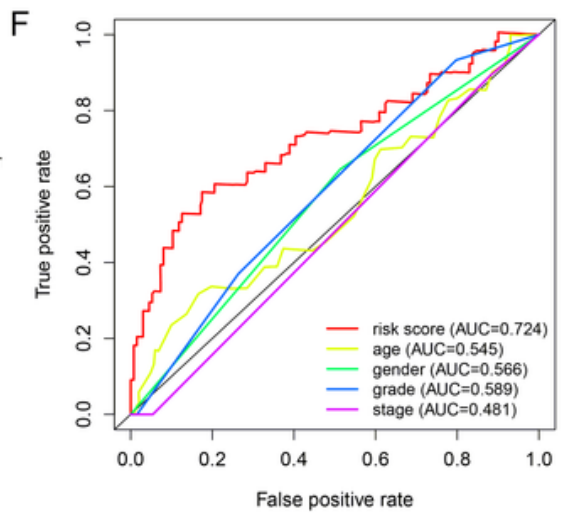

G

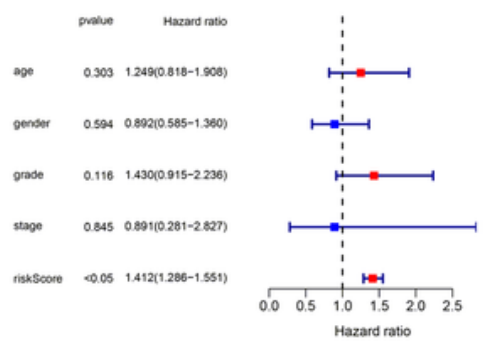

$\mathrm{H}$

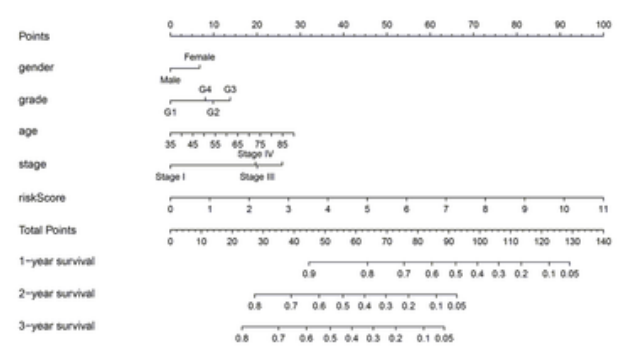

\section{Figure 3}

(A-D).Different levels of risk scores in pantients with pancreatic cancer stratified by age,gender,grade and stage. (E).A strip chart of the associations between risk score and clinicopathological features in the TCGA dataset. (F). The receiver operating characteristic (ROC) curves for the risk score,age,gender,grade 
and stage in the TCGA dataset. (G). Results of the univariate Cox regression analysis regarding OS in the TCGA cohort.Risk score was the only independent prognostic parameter. H.Nomogram based on risk score,age,gender,grade and stage.

A

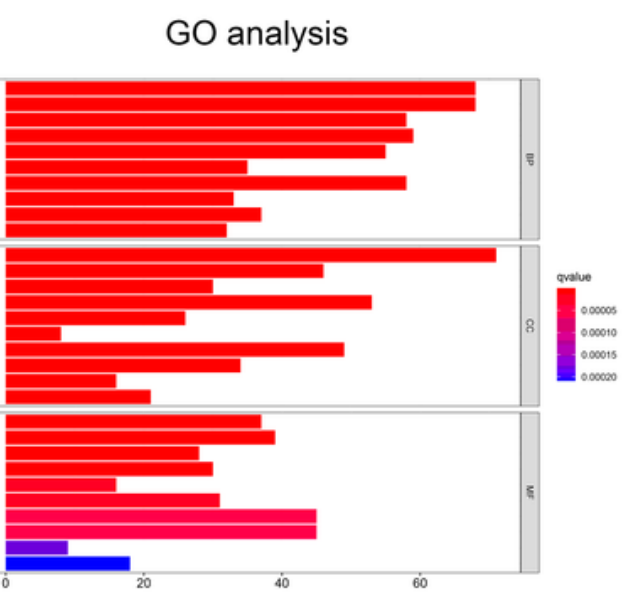

B

KEGG analysis

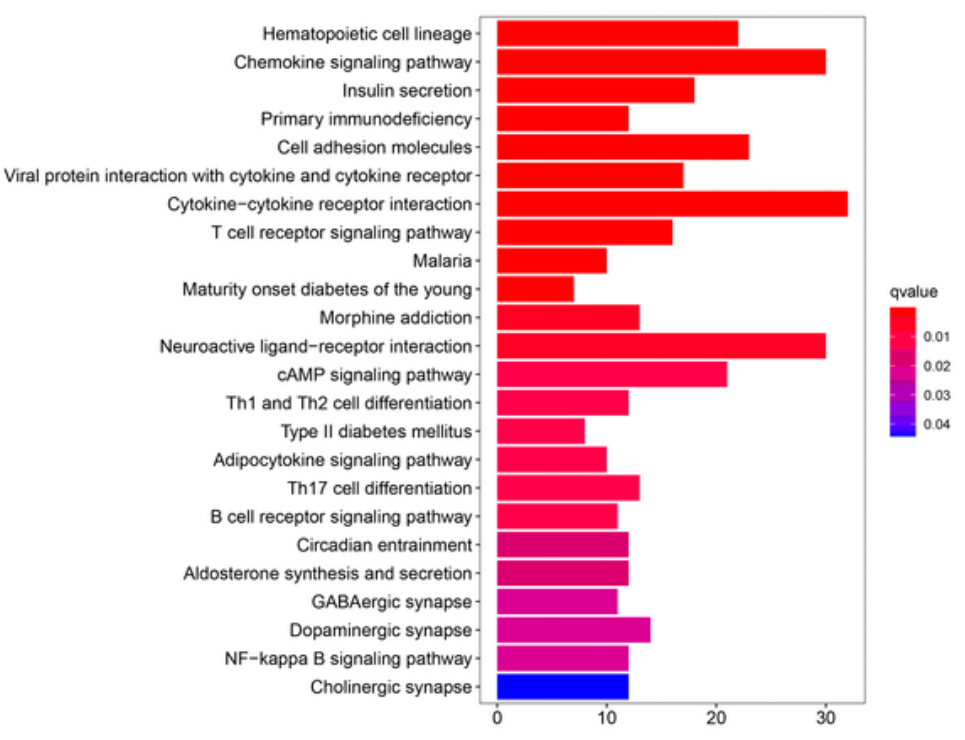

Figure 4

(A).The GO analysis of DEGs between the high- and low-risk groups. (B).The KEGG analysis of DEGs between the high- and low-risk groups. 

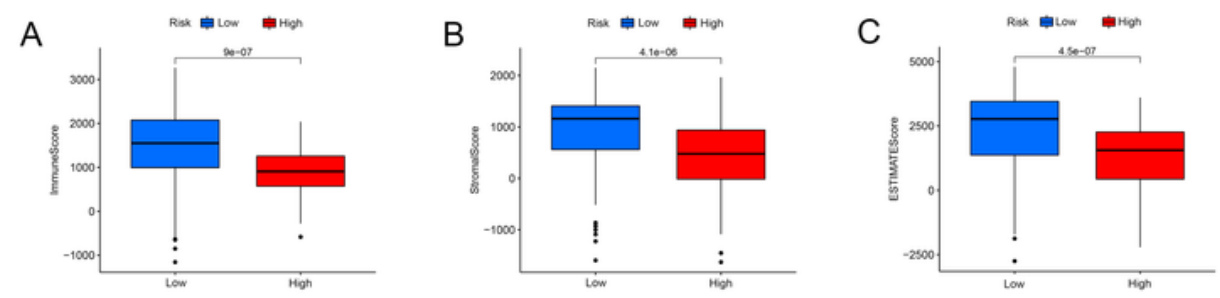

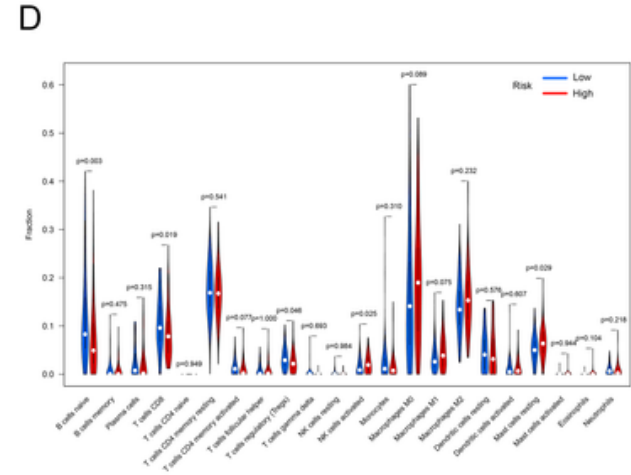

$\mathrm{F}$
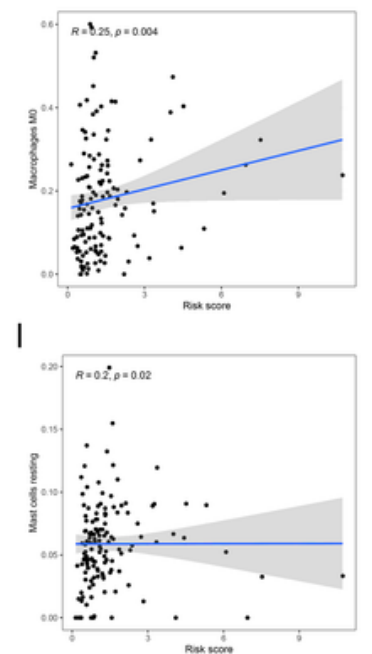

$\mathrm{L}$

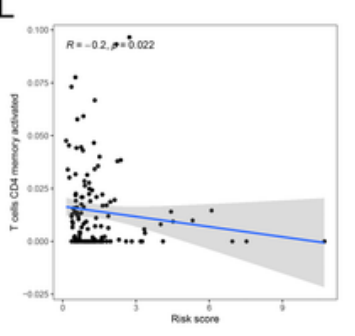

G

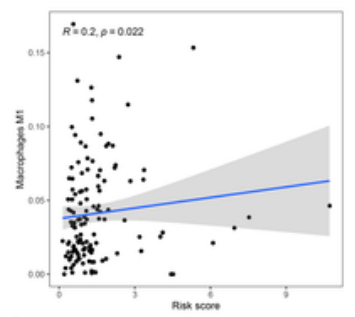

$\mathrm{J}$

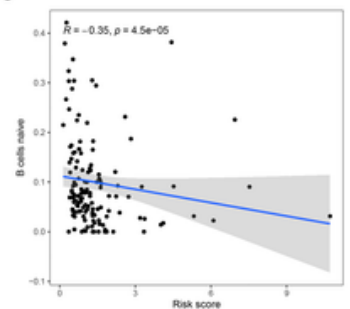

M

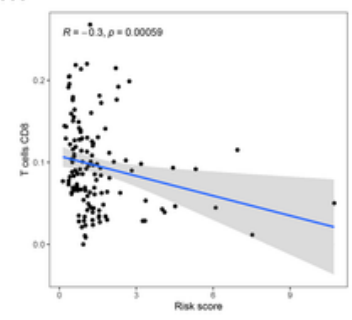

E

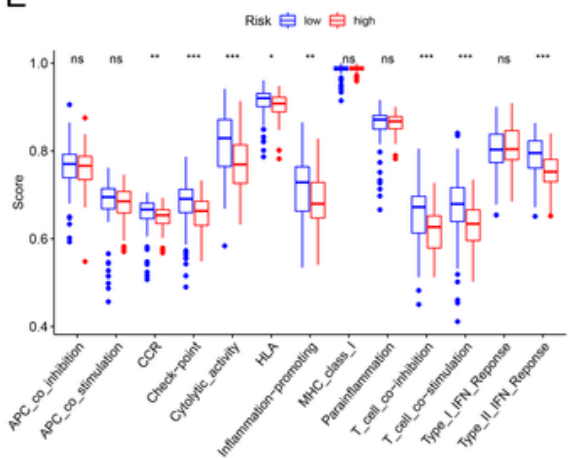

$\mathrm{H}$

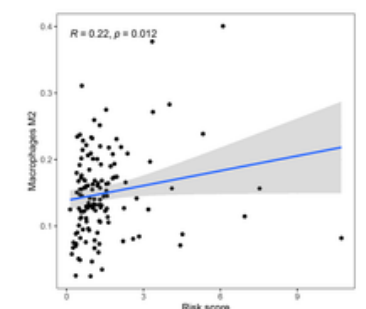

K

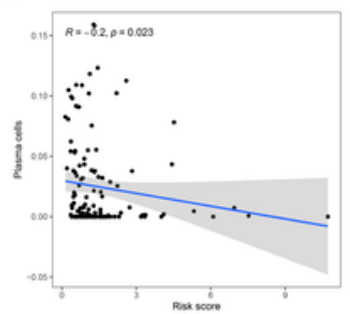

$\mathrm{N}$

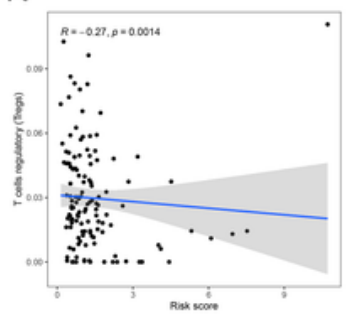

\section{Figure 5}

(A-C).Different expression of immune score,stromal score and ESTIMATE score in the high- and low-risk groups. (D).Violin plot visualizing the differentially infiltrated immune cells. (E). The ssGSEA scores of 13 immune-related functions between different risk groups in the TCGA cohort. (F-N). Relationships between the FRLS and infiltration abundances of nine types of immune cells. The correlation was performed by Pearson correlation analysis. (F).Macrophages M0; (G).Macrophages M1; (H).Macrophages M2; (I).Mast 
cells resting; (J).B cells naive; (K).Plasma cells;(L).T cells CD4 memory activated; (M).T cells CD8; (N).T cells regulatory. Treg, regulatory T cell; APC,antigen presenting cell; CCR, cytokine-cytokine receptor; HLA, human leukocyte antigen; MHC, major histocompatibility complex; IFN, immune interferon; ns, not significant; $* \star \star P<0.001 ; * * \mathrm{P}<0.01 ; * \mathrm{P}<0.05$.
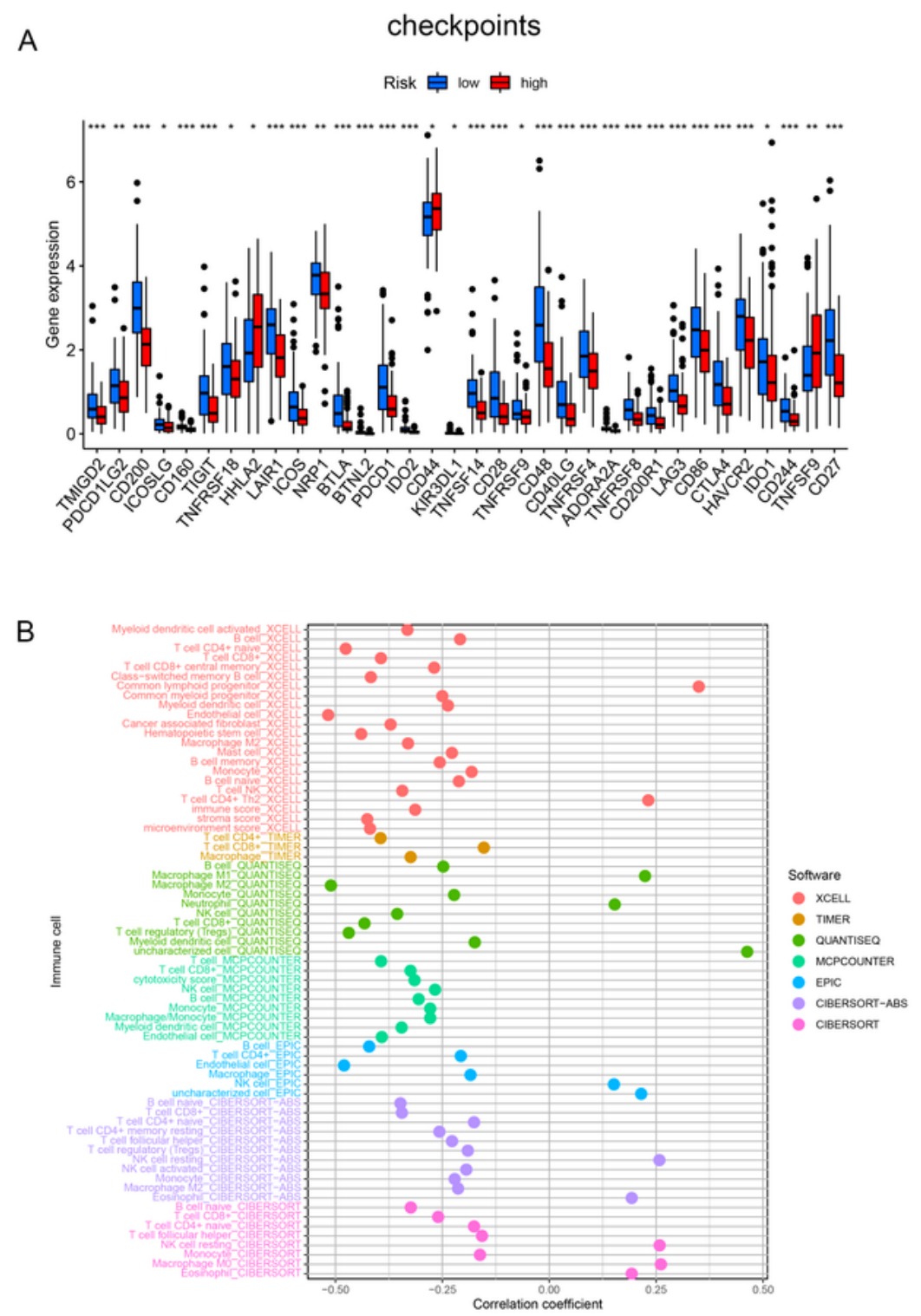

Figure 6 
(A).Differences expression of thirty-four immune checkpoints in the two risk groups. (B).Estimation of tumor-infiltrating immune cells based on XCELL,TIMER,QUANTISEQ,MCPOUNTER,EPIC,CIBERSORTABSand CIBERSORT algorithms. ${ }^{* \star *} \mathrm{P}<0.001 ; * \star \mathrm{P}<0.01 ; * \mathrm{P}<0.05$. 